\title{
Volterra composition operators from generalized weighted weighted Bergman spaces to $\mu$-Bloch spaces
}

\author{
Xiangling Zhu
}

(Communicated by Miroslav Engliš)

2000 Mathematics Subject Classification. Primary 47B35, Secondary $30 \mathrm{H} 05$.

Keywords and phrases. Volterra composition operator, generalized weighted Bergman space, $\mu$-Bloch space.

Abstract. Let $\varphi$ be a holomorphic self-map and $g$ be a fixed holomorphic function on the unit ball $B$. The boundedness and compactness of the operator

$$
T_{g, \varphi} f(z)=\int_{0}^{1} f(\varphi(t z)) \Re g(t z) \frac{d t}{t}
$$

from the generalized weighted Bergman space into the $\mu$-Bloch space are studied in this paper.

\section{Introduction}

Let $B$ be the unit ball of $\mathbb{C}^{n}$. Let $z=\left(z_{1}, \ldots, z_{n}\right)$ and $w=\left(w_{1}, \ldots, w_{n}\right)$ be points in $\mathbb{C}^{n}$, we write

$$
\langle z, w\rangle=z_{1} \bar{w}_{1}+\cdots+z_{n} \bar{w}_{n},|z|=\sqrt{\left|z_{1}\right|^{2}+\cdots+\left|z_{n}\right|^{2}} .
$$

Thus $B=\left\{z \in \mathbb{C}^{n}:|z|<1\right\}$. Let $d v$ be the normalized Lebesgue measure of $B$, i.e. $v(B)=1$. Let $H(B)$ be the space of all holomorphic functions 
on $B$. For $f \in H(B)$, let $\Re f(z)=\sum_{j=1}^{n} z_{j} \frac{\partial f}{\partial z_{j}}(z)$ represent the radial derivative of $f \in H(B)$. We write $\Re^{m} f=\Re\left(\Re^{m-1} f\right)$.

A positive continuous function $\mu$ on $[0,1)$ is called normal, if there exist positive numbers $s$ and $t, 0<s<t$, and $\delta \in[0,1)$ such that

$$
\begin{aligned}
& \frac{\mu(r)}{(1-r)^{s}} \text { is decreasing on }[\delta, 1) \text { and } \lim _{r \rightarrow 1} \frac{\mu(r)}{(1-r)^{s}}=0 ; \\
& \frac{\mu(r)}{(1-r)^{t}} \text { is increasing on }[\delta, 1) \text { and } \lim _{r \rightarrow 1} \frac{\mu(r)}{(1-r)^{t}}=\infty
\end{aligned}
$$

(see, e.g. [4]).

Let $\mu$ be a normal function on $[0,1)$. The $\mu$-Bloch space, denoted by $\mathcal{B}_{\mu}=\mathcal{B}_{\mu}(B)$, is the set of all $f \in H(B)$ such that

$$
b_{\mu}(f)=\sup _{z \in B} \mu(|z|)|\Re f(z)|<\infty .
$$

$\mathcal{B}_{\mu}$ is a Banach space with the norm $\|f\|_{\mathcal{B}_{\mu}}=|f(0)|+b_{\mu}(f)$. Let $\mathcal{B}_{\mu, 0}$ denote the subspace of $\mathcal{B}_{\mu}$ consisting of those $f \in \mathcal{B}_{\mu}$ for which

$$
\lim _{|z| \rightarrow 1} \mu(|z|)|\Re f(z)|=0 .
$$

We call $\mathcal{B}_{\mu, 0}$ the little $\mu$-Bloch space. When $\mu(r)=1-r^{2}$ and $\mu(r)=$ $\left(1-r^{2}\right)^{1-\beta}(0<\beta<1)$, the induced spaces $\mathcal{B}_{\mu}$ are the Bloch spaces and the Lipschitz type spaces, respectively.

For any $p>0$ and $\alpha \in \mathbb{R}$, let $N$ be the smallest nonnegative integer such that $p N+\alpha>-1$. We say that an $f \in H(B)$ belongs to the generalized weighted Bergman space $A_{\alpha}^{p}$, if

$$
\|f\|_{A_{\alpha}^{p}}=|f(0)|+\left[\int_{B}\left|\Re^{N} f(z)\right|^{p}\left(1-|z|^{2}\right)^{p N+\alpha} d v(z)\right]^{1 / p}<\infty .
$$

The generalized weighted Bergman space $A_{\alpha}^{p}$ is introduced by Zhao and Zhu (see, e.g., [15]). This space covers the traditional weighted Bergman space $(a>-1)$, the Besov space, the Hardy space $H^{2}$ and the so-called Arveson space. For example, the space $A_{0}^{p}$ is the classical Bergman space; the space $A_{-n}^{2}$ is the so-called Arveson space; the space $A_{-(n+1)}^{p}$ is the Besov space. See $[15,16]$ for some basic facts on the weighted Bergman space.

Let $\varphi$ be a holomorphic self-map of $B$. The composition operator $C_{\varphi}$ is defined by

$$
\left(C_{\varphi} f\right)(z)=(f \circ \varphi)(z), f \in H(B)
$$


The book [2] contains much information on this topic.

Suppose that $g: B \rightarrow \mathbb{C}^{1}$ is a holomorphic map, the extended Cesàro operator, which was introduced in [4], is defined as following

$$
T_{g} f(z)=\int_{0}^{1} f(t z) \frac{d g(t z)}{d t}=\int_{0}^{1} f(t z) \Re g(t z) \frac{d t}{t}, \quad f \in H(B), z \in B .
$$

This operator is also called the Riemann-Stieltjes operator(see, e.g. [14]). See $[1,4,5,6,8,9,10,13,14]$ for more information of the operator $T_{g}$ on various spaces in the unit ball.

Motivated by the definition of operators $C_{\varphi}$ and $T_{g}$, we define a more general operator

$$
T_{g, \varphi} f(z)=\int_{0}^{1} f(\varphi(t z)) \Re g(t z) \frac{d t}{t}, \quad f \in H(B), z \in B .
$$

The operator $T_{g, \varphi}$ will be called the Volterra composition operator. In the setting of the unit disk $D$, this operator has the following form

$$
T_{g, \varphi} f(z)=\int_{0}^{z}(f \circ \varphi)(\xi) g^{\prime}(\xi) d \xi, \quad f \in H(D), z \in D,
$$

which was first studied in [7]. To the best of our knowledge, the operator $T_{g, \varphi}$ in the unit ball is studied in the present paper for the first time.

In this paper we study the boundedness and compactness of Volterra composition operators $T_{g, \varphi}$ from the generalized weighted Bergman space into $\mathcal{B}_{\mu}$ and $\mathcal{B}_{\mu, 0}$. As some corollaries, we obtain characterizations of the extended Cesàro operator $T_{g}$ from the generalized weighted Bergman space into $\mathcal{B}_{\mu}$ and $\mathcal{B}_{\mu, 0}$.

Throughout the paper, constants are denoted by $C$, they are positive and may differ from one occurrence to the other.

\section{Main results and proofs}

In this section we give our main results and proofs. We will consider three cases: $n+1+\alpha>0, n+1+\alpha=0$ and $n+1+\alpha<0$. Before we formulate our main results, we state several auxiliary results which will be used in the proofs. They are incorporated in the lemmas which follows.

Lemma 1. [15] (i) Suppose $p>0$ and $\alpha+n+1>0$. Then there exists a constant $C>0$ such that

$$
|f(z)| \leq \frac{C\|f\|_{A_{\alpha}^{p}}}{\left(1-|z|^{2}\right)^{\frac{n+\alpha+1}{p}}}
$$


for all $f \in A_{\alpha}^{p}$ and $z \in B$.

(ii) Suppose $p>0$ and $\alpha+n+1<0$ or $0<p \leq 1$ and $\alpha+n+1=0$. Then every function in $A_{\alpha}^{p}$ is continuous on the closed unit ball and so is bounded.

(iii) Suppose $p>1,1 / p+1 / q=1$ and $\alpha+n+1=0$. Then there exists a constant $C>0$ such that

$$
|f(z)| \leq C\left[\ln \frac{2}{1-|z|^{2}}\right]^{1 / q}
$$

for all $f \in A_{\alpha}^{p}$ and $z \in B$.

Lemma 2. A closed set $K$ in $\mathcal{B}_{\mu, 0}$ is compact if and only if it is bounded and satisfies

$$
\lim _{|z| \rightarrow 1} \sup _{f \in K} \mu(|z|)|\Re f(z)|=0 .
$$

Proof. The proof is similar to the proof of Lemma 1 in [11]. We omit the details.

The following criterion for compactness follows from standard arguments similar to those outlined in Proposition 3.11 of [2]. We omit the details of the proof.

Lemma 3. Assume that $p>0, \alpha$ is a real number, $g \in H(B), \varphi$ is a holomorphic self-map of $B$ and $\mu$ is a normal function on $[0,1)$. Then $T_{g, \varphi}: A_{\alpha}^{p} \rightarrow \mathcal{B}_{\mu}$ is compact if and only if $T_{g, \varphi}: A_{\alpha}^{p} \rightarrow \mathcal{B}_{\mu}$ is bounded and for any bounded sequence $\left(f_{k}\right)_{k \in \mathbb{N}}$ in $A_{\alpha}^{p}$ which converges to zero uniformly on compact subsets of $B$ as $k \rightarrow \infty$, we have $\left\|T_{g, \varphi} f_{k}\right\|_{\mathcal{B}_{\mu}} \rightarrow 0$ as $k \rightarrow \infty$.

Especially, when $p>0$ and $\alpha+n+1<0$, we need the following criterion for compactness follows from arguments similar to those in Lemma 3.7 of [12].

Lemma 4. Let $p>0$ and $\alpha+n+1<0$. Let $T$ be a bounded linear operator from $A_{\alpha}^{p}$ into a normed linear space $Y$. Then $T$ is compact if and only if $\left\|T f_{k}\right\|_{Y} \rightarrow 0$ whenever $\left(f_{k}\right)$ is a norm-bounded sequence in $A_{\alpha}^{p}$ that converges to 0 uniformly on $\bar{B}$.

Proof. The necessity is obvious. Now we prove the sufficiency part. Suppose that $T$ is not compact. Then there is a bounded sequence $\left(g_{k}\right)$ in $A_{\alpha}^{p}$ such that $\left(T g_{k}\right)$ has no convergent subsequence. Note that when $p>0$ and $\alpha+n+1<0, A_{\alpha}^{p}$ are indeed Lipschitz continuous (see Theorem 66 of [15]). Similarly to the proof of Lemma 3.6 of [12], we see that every bounded sequence in $A_{\alpha}^{p}$ has a subsequence that converges uniformly on $\bar{B}$ by Lemma 1 and Arzela-Ascoli Theorem. Hence $\left(g_{k}\right)$ has a subsequence $\left(f_{k}\right)$ such that $f_{k} \rightarrow f$ uniformly on $\bar{B}$. By Fatou's lemma we see that 
$f \in A_{\alpha}^{p}$. The sequence $\left(f_{k}-f\right)$ is bounded in $A_{\alpha}^{p}$ and converges to 0 uniformly on $\bar{B}$. By assumption $\left\|T f_{k}-T f\right\|_{Y} \rightarrow 0$ as $k \rightarrow \infty$. This implies that the subsequence $\left(T f_{k}\right)$ of $\left(T g_{k}\right)$ converges in $Y$ (to $T f$ ), a contradiction.

\subsection{Case $n+1+\alpha>0$.}

Theorem 1. Assume that $p>0, \alpha$ is a real number such that $n+\alpha+1>0, g \in H(B), \varphi$ is a holomorphic self-map of $B$ and $\mu$ is a normal function on $[0,1)$. Then $T_{g, \varphi}: A_{\alpha}^{p} \rightarrow \mathcal{B}_{\mu}$ is bounded if and only if

$$
M:=\sup _{z \in B} \frac{\mu(|z|)|\Re g(z)|}{\left(1-|\varphi(z)|^{2}\right)^{\frac{n+1+\alpha}{p}}}<\infty .
$$

Proof. Suppose that (2) holds. A calculation with (1) gives the following fundamental and useful formula(see, e.g. [4])

$$
\Re\left[T_{g, \varphi}(f)\right](z)=f(\varphi(z)) \Re g(z) .
$$

Then for arbitrary $z \in B$ and $f \in A_{\alpha}^{p}$, by Lemma 1 we have

$$
\begin{aligned}
\mu(|z|)\left|\Re\left(T_{g, \varphi} f\right)(z)\right| & =\mu(|z|)|f(\varphi(z))||\Re g(z)| \\
& \leq C\|f\|_{A_{\alpha}^{p}} \frac{\mu(|z|)|\Re g(z)|}{\left(1-|\varphi(z)|^{2}\right)^{\frac{n+1+\alpha}{p}}} .
\end{aligned}
$$

Using the condition (2), the boundedness of the operator $T_{g, \varphi}: A_{\alpha}^{p} \rightarrow \mathcal{B}_{\mu}$ follows by taking the supremum in (3) over $B$.

Conversely, suppose that $T_{g, \varphi}: A_{\alpha}^{p} \rightarrow \mathcal{B}_{\mu}$ is bounded. Assume that

$$
t>n \max \left(1, \frac{1}{p}\right)+\frac{\alpha+1}{p} .
$$

For $a \in B$, set

$$
f_{a}(z)=\frac{\left(1-|a|^{2}\right)^{t-\frac{n+1+\alpha}{p}}}{(1-\langle z, a\rangle)^{t}} .
$$

Then from Theorem 32 of [15] we see that $f_{a} \in A_{\alpha}^{p}$ and $\sup _{a \in B}\left\|f_{a}\right\|_{A_{\alpha}^{p}<}$ $\infty$. Therefore

$$
\begin{aligned}
C\left\|T_{g, \varphi}\right\|_{A_{\alpha}^{p} \rightarrow \mathcal{B}_{\mu}} & \geq\left\|T_{g, \varphi} f_{\varphi(b)}\right\|_{\mathcal{B}_{\mu}} \geq \sup _{z \in B} \mu(|z|)\left|\Re\left(T_{g, \varphi} f_{\varphi(b)}\right)(z)\right| \\
& \geq \frac{\mu(|b|)|\Re g(b)|}{\left(1-|\varphi(b)|^{2}\right)^{\frac{n+1+\alpha}{p}}}
\end{aligned}
$$


from which we get (2). This completes the proof of Theorem 1 .

Theorem 2. Assume that $p>0, \alpha$ is a real number such that $n+\alpha+1>0, g \in H(B), \varphi$ is a holomorphic self-map of $B$ and $\mu$ is a normal function on $[0,1)$. Then $T_{g, \varphi}: A_{\alpha}^{p} \rightarrow \mathcal{B}_{\mu}$ is compact if and only if $g \in \mathcal{B}_{\mu}$ and

$$
\lim _{|\varphi(z)| \rightarrow 1} \frac{\mu(|z|)|\Re g(z)|}{\left(1-|\varphi(z)|^{2}\right)^{\frac{n+1+\alpha}{p}}}=0 .
$$

Proof. Suppose that $g \in \mathcal{B}_{\mu}$ and (6) holds. From $g \in \mathcal{B}_{\mu}$ and (6), it is easy to see that (2) holds. Hence $T_{g, \varphi}: A_{\alpha}^{p} \rightarrow \mathcal{B}_{\mu}$ is bounded by Theorem 1. From (6), for given $\varepsilon>0$, there is a constant $\delta \in(0,1)$, such that

$$
\sup _{\{z \in B: \delta<|\varphi(z)|<1\}} \frac{\mu(|z|)|\Re g(z)|}{\left(1-|\varphi(z)|^{2}\right)^{\frac{n+1+\alpha}{p}}}<\varepsilon .
$$

Let $\left(f_{k}\right)_{k \in \mathbb{N}}$ be a bounded sequence in $A_{\alpha}^{p}$ such that $f_{k} \rightarrow 0$ uniformly on compact subsets of $B$ as $k \rightarrow \infty$. Let $G=\{w \in B:|w| \leq \delta\}$. From the fact that $g \in \mathcal{B}_{\mu}$ and (7), we have

$$
\begin{aligned}
& \left\|T_{g, \varphi} f_{k}\right\|_{\mathcal{B}_{\mu}}=\sup _{z \in B} \mu(|z|)\left|f_{k}(\varphi(z)) \Re g(z)\right| \\
= & \left(\sup _{\{z \in B:|\varphi(z)| \leq \delta\}}+\sup _{\{z \in B: \delta<|\varphi(z)|<1\}}\right) \mu(|z|)|\Re g(z)|\left|f_{k}(\varphi(z))\right| \\
= & \|g\|_{\mathcal{B}_{\mu}} \sup _{w \in G}\left|f_{k}(w)\right|+C\left\|f_{k}\right\|_{A_{\alpha}^{p}} \sup _{\{z \in B: \delta<|\varphi(z)|<1\}} \frac{\mu(|z|)|\Re g(z)|}{\left(1-|\varphi(z)|^{2}\right)^{\frac{n+1+\alpha}{p}}} \\
(8) \leq & \|g\|_{\mathcal{B}_{\mu}} \sup _{w \in G}\left|f_{k}(w)\right|+C \varepsilon .
\end{aligned}
$$

Observe that $G$ is a compact subset of $B$, then it gives $\lim _{k \rightarrow \infty} \sup _{w \in G}\left|f_{k}(w)\right|=$ 0 . Using this fact and letting $k \rightarrow \infty$ in (8), we obtain $\lim _{\sup _{k \rightarrow \infty}}\left\|T_{g, \varphi} f_{k}\right\|_{\mathcal{B}_{\mu}} \leq$ $C \varepsilon$. Since $\varepsilon$ is an arbitrary positive number, we obtain $\lim _{\sup } \sup _{k \rightarrow \infty}\left\|T_{g, \varphi} f_{k}\right\|_{\mathcal{B}_{\mu}}=$ 0 . Employing Lemma 3, we get that $T_{g, \varphi}: A_{\alpha}^{p} \rightarrow \mathcal{B}_{\mu}$ is compact.

Conversely, suppose that $T_{g, \varphi}: A_{\alpha}^{p} \rightarrow \mathcal{B}_{\mu}$ is compact, then $T_{g, \varphi}: A_{\alpha}^{p} \rightarrow$ $\mathcal{B}_{\mu}$ is bounded. It follows from the proof of Theorem 1 that $g \in \mathcal{B}_{\mu}$. Let $\left(z_{k}\right)_{k \in \mathbb{N}}$ be a sequence in $B$ such that $\left|\varphi\left(z_{k}\right)\right| \rightarrow 1$ as $k \rightarrow \infty$. Set

$$
f_{k}(z)=\frac{\left(1-\left|\varphi\left(z_{k}\right)\right|^{2}\right)^{t-\frac{n+\alpha+1}{p}}}{\left(1-\left\langle z, \varphi\left(z_{k}\right)\right\rangle\right)^{t}}, k \in \mathbb{N},
$$

where $t$ satisfies (4). From Theorem 32 of [15] we see that $\left(f_{k}\right)_{k \in \mathbb{N}}$ is a bounded sequence in $A_{\alpha}^{p}$. Moreover, it is easy to see that $f_{k}$ converges to 
zero uniformly on compact subsects of $B$. In view of Lemma 3 it follows that

$$
\limsup _{k \rightarrow \infty}\left\|T_{g, \varphi} f_{k}\right\|_{\mathcal{B}_{\mu}}=0
$$

In addition, we have

$$
\left\|T_{g, \varphi} f_{k}\right\|_{\mathcal{B}_{\mu}}=\sup _{z \in B} \mu(|z|)\left|\Re\left(T_{g, \varphi} f_{k}\right)(z)\right| \geq \frac{\mu\left(\left|z_{k}\right|\right)\left|\Re g\left(z_{k}\right)\right|}{\left(1-\left|\varphi\left(z_{k}\right)\right|^{2}\right)^{\frac{n+1+\alpha}{p}}} .
$$

Combining (9) with (10) we get the desired result. The proof is completed.

Theorem 3. Assume that $p>0, \alpha$ is a real number such that $n+\alpha+1>0, g \in H(B), \varphi$ is a holomorphic self-map of $B$ and $\mu$ is a normal function on $[0,1)$. Then $T_{g, \varphi}: A_{\alpha}^{p} \rightarrow \mathcal{B}_{\mu, 0}$ is bounded if and only if $T_{g, \varphi}: A_{\alpha}^{p} \rightarrow \mathcal{B}_{\mu}$ is bounded and $g \in \mathcal{B}_{\mu, 0}$.

Proof. Suppose that $T_{g, \varphi}: A_{\alpha}^{p} \rightarrow \mathcal{B}_{\mu, 0}$ is bounded. Then it is clear that $T_{g, \varphi}: A_{\alpha}^{p} \rightarrow \mathcal{B}_{\mu}$ is bounded. Taking $f(z)=1$ and employing the boundedness of $T_{g, \varphi}: A_{\alpha}^{p} \rightarrow \mathcal{B}_{\mu, 0}$, we see that $g \in \mathcal{B}_{\mu, 0}$.

Conversely, suppose that $T_{g, \varphi}: A_{\alpha}^{p} \rightarrow \mathcal{B}_{\mu}$ is bounded and $g \in \mathcal{B}_{\mu, 0}$. Suppose that $f \in A_{\alpha}^{p}$ with $\|f\|_{A_{\alpha}^{p}} \leq L$, using polynomial approximations we obtain (see, e.g., [15])

$$
\lim _{|z| \rightarrow 1}\left(1-|z|^{2}\right)^{\frac{n+1+\alpha}{p}}|f(z)|=0 .
$$

From the above equality and $g \in \mathcal{B}_{\mu, 0}$, for every $\varepsilon>0$, there exists a $\delta \in(0,1)$ such that when $\delta<|z|<1$,

$$
\left(1-|z|^{2}\right)^{\frac{n+1+\alpha}{p}}|f(z)|<\varepsilon / M
$$

and

$$
\mu(|z|)|\Re g(z)|<\frac{\varepsilon\left(1-\delta^{2}\right)^{\frac{n+1+\alpha}{p}}}{L},
$$

where $M$ is defined in (2). Therefore if $\delta<|z|<1$ and $\delta<|\varphi(z)|<1$, from (2) and (11) we have

$$
\begin{aligned}
\mu(|z|)\left|\Re\left(T_{g, \varphi} f\right)(z)\right| & =\frac{\mu(|z|)|\Re g(z)|}{\left(1-|\varphi(z)|^{2}\right)^{\frac{n+1+\alpha}{p}}}\left(1-|\varphi(z)|^{2}\right)^{\frac{n+1+\alpha}{p}}|f(\varphi(z))| \\
& \leq M\left(1-|\varphi(z)|^{2}\right)^{\frac{n+1+\alpha}{p}}|f(\varphi(z))|<\varepsilon .
\end{aligned}
$$


If $\delta<|z|<1$ and $|\varphi(z)| \leq \delta$, using Lemma 1 and (12) we have

$$
\begin{aligned}
\mu(|z|)\left|\Re\left(T_{g, \varphi} f\right)(z)\right| & =\frac{\mu(|z|)|\Re g(z)|}{\left(1-|\varphi(z)|^{2}\right)^{\frac{n+1+\alpha}{p}}}\left(1-|\varphi(z)|^{2}\right)^{\frac{n+1+\alpha}{p}}|f(\varphi(z))| \\
& \leq C\|f\|_{A_{\alpha}^{p}} \frac{\mu(|z|)|\Re g(z)|}{\left(1-|\varphi(z)|^{2}\right)^{\frac{n+1+\alpha}{p}}} \\
& \leq \frac{C\|f\|_{A_{\alpha}^{p}}}{\left(1-\delta^{2}\right) \frac{n+1+\alpha}{p}} \mu(|z|)|\Re g(z)|<C \varepsilon .
\end{aligned}
$$

Combining (13) with (14) we get that $T_{g, \varphi} f \in \mathcal{B}_{\mu, 0}$. Since $f$ is arbitrary we see that $T_{g, \varphi}\left(A_{\alpha}^{p}\right) \subset \mathcal{B}_{\mu, 0}$, which together with the boundedness of $T_{g, \varphi}: A_{\alpha}^{p} \rightarrow \mathcal{B}_{\mu}$, we get the desired result. This completes the proof of the theorem.

Theorem 4. Assume that $p>0, \alpha$ is a real number such that $n+\alpha+1>0, g \in H(B), \varphi$ is a holomorphic self-map of $B$ and $\mu$ is a normal function on $[0,1)$. Then $T_{g, \varphi}: A_{\alpha}^{p} \rightarrow \mathcal{B}_{\mu, 0}$ is compact if and only if

$$
\lim _{|z| \rightarrow 1} \frac{\mu(|z|)|\Re g(z)|}{\left(1-|\varphi(z)|^{2}\right)^{\frac{n+1+\alpha}{p}}}=0
$$

Proof. Suppose that $T_{g, \varphi}: A_{\alpha}^{p} \rightarrow \mathcal{B}_{\mu, 0}$ is compact. Then $T_{g, \varphi}: A_{\alpha}^{p} \rightarrow$ $\mathcal{B}_{\mu, 0}$ is bounded and $T_{g, \varphi}: A_{\alpha}^{p} \rightarrow \mathcal{B}_{\mu}$ is compact. By Theorems 2 and 3 we obtain

$$
\lim _{|\varphi(z)| \rightarrow 1} \frac{\mu(|z|)|\Re g(z)|}{\left(1-|\varphi(z)|^{2}\right)^{\frac{n+1+\alpha}{p}}}=0 .
$$

and

$$
\lim _{|z| \rightarrow 1} \mu(|z|)|\Re g(z)|=0,
$$

By (16), for every $\varepsilon>0$, there exists a $\delta \in(0,1)$,

$$
\frac{\mu(|z|)|\Re g(z)|}{\left(1-|\varphi(z)|^{2}\right)^{\frac{n+1+\alpha}{p}}}<\varepsilon
$$

when $\delta<|\varphi(z)|<1$. By (17), for the above $\varepsilon$, there exists $r \in(0,1)$,

$$
\mu(|z|)|\Re g(z)| \leq \varepsilon\left(1-|\delta|^{2}\right)^{\frac{n+1+\alpha}{p}}
$$

when $r<|z|<1$. 
Therefore, when $r<|z|<1$ and $\delta<|\varphi(z)|<1$, we have that

$$
\frac{\mu(|z|)|\Re g(z)|}{\left(1-|\varphi(z)|^{2}\right)^{\frac{n+1+\alpha}{p}}}<\varepsilon .
$$

If $|\varphi(z)| \leq \delta$ and $r<|z|<1$, we obtain

$$
\frac{\mu(|z|)|\Re g(z)|}{\left(1-|\varphi(z)|^{2}\right)^{\frac{n+1+\alpha}{p}}} \leq \frac{1}{\left(1-|\delta|^{2}\right)^{\frac{n+1+\alpha}{p}}} \mu(|z|)|\Re g(z)|<\varepsilon .
$$

Combing (18) with (19) we get (15) as desired.

Conversely, suppose that (15) holds. It follows from Lemma 2 that $T_{g, \varphi}: A_{\alpha}^{p} \rightarrow \mathcal{B}_{\mu, 0}$ is compact if and only if

$$
\lim _{|z| \rightarrow 1} \sup _{\|f\|_{A_{\alpha}^{p} \leq 1}} \mu(|z|)\left|\Re\left(T_{g, \varphi} f\right)(z)\right|=0 .
$$

For any $f \in A_{\alpha}^{p}$ with $\|f\|_{A_{\alpha}^{p}} \leq 1$, by (3) we have

$$
\mu(|z|)\left|\Re\left(T_{g, \varphi} f\right)(z)\right| \leq C\|f\|_{A_{\alpha}^{p}} \frac{\mu(|z|)|\Re g(z)|}{\left(1-|\varphi(z)|^{2}\right)^{\frac{n+1+\alpha}{p}}} .
$$

Using (15) we get

$$
\lim _{|z| \rightarrow 1} \sup _{\|f\|_{A_{\alpha}^{p} \leq 1}} \mu(|z|)\left|\Re\left(T_{g, \varphi} f\right)(z)\right| \leq C \lim _{|z| \rightarrow 1} \frac{\mu(|z|)|\Re g(z)|}{\left(1-|\varphi(z)|^{2}\right)^{\frac{n+1+\alpha}{p}}}=0,
$$

as desired. This completes the proof of the theorem.

Let $\varphi(z)=z, \mu(r)=\left(1-r^{2}\right)^{\beta}$. From Theorems 1-4 we have the following result (see $[8,9]$ for the case of $\alpha>-1$ ).

Corollary 1. Assume that $p>0, \alpha$ is a real number such that $n+\alpha+1>0, \frac{n+1+\alpha}{p} \leq \beta<\infty$ and $g \in H(B)$. Then the following statements hold.

(i) $T_{g}: A_{\alpha}^{p} \rightarrow \mathcal{B}^{\beta}$ is bounded if and only if $T_{g}: A_{\alpha}^{p} \rightarrow \mathcal{B}_{0}^{\beta}$ is bounded if and only if

$$
\sup _{z \in B}\left(1-|z|^{2}\right)^{\beta-\frac{n+1+\alpha}{p}}|\Re g(z)|<\infty ;
$$

(ii) $T_{g}: A_{\alpha}^{p} \rightarrow \mathcal{B}^{\beta}$ is compact if and only if $T_{g}: A_{\alpha}^{p} \rightarrow \mathcal{B}_{0}^{\beta}$ is compact if and only if

$$
\lim _{|z| \rightarrow 1}\left(1-|z|^{2}\right)^{\beta-\frac{n+1+\alpha}{p}}|\Re g(z)|=0 .
$$


2.2 Case $n+1+\alpha=0$. First, we consider the case $p>1$.

Theorem 5. Assume that $p>1,1 / p+1 / q=1$ and $n+1+\alpha=0$, $g \in H(B), \varphi$ is a holomorphic self-map of $B$ and $\mu$ is a normal function on $[0,1)$. Then $T_{g, \varphi}: A_{\alpha}^{p} \rightarrow \mathcal{B}_{\mu}$ is bounded if and only if

$$
M_{3}:=\sup _{z \in B} \mu(|z|)|\Re g(z)|\left(\ln \frac{2}{1-|\varphi(z)|^{2}}\right)^{1 / q}<\infty .
$$

Proof. Suppose that (21) holds. Then for arbitrary $z \in B$ and $f \in A_{\alpha}^{p}$, by Lemma 1 we have

$$
\begin{aligned}
& \mu(|z|)\left|\Re\left(T_{g, \varphi} f\right)(z)\right|=\mu(|z|)|f(\varphi(z))||\Re g(z)|
\end{aligned}
$$

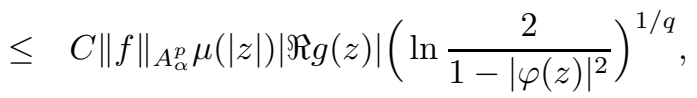

from which we see that $T_{g, \varphi}: A_{\alpha}^{p} \rightarrow \mathcal{B}_{\mu}$ is bounded.

Conversely, suppose that $T_{g, \varphi}: A_{\alpha}^{p} \rightarrow \mathcal{B}_{\mu}$ is bounded. For $a \in B$, set

$$
f_{a}(z)=\left(\ln \frac{2}{1-|a|^{2}}\right)^{-1 / p}\left(\ln \frac{2}{1-\langle z, a\rangle}\right) .
$$

Using Theorem 1.12 of [16], it is easy to check that $f_{a} \in A_{-(n+1)}^{p}$. Therefore

$$
\begin{aligned}
C\left\|T_{g, \varphi}\right\|_{A_{\alpha}^{p} \rightarrow \mathcal{B}_{\mu}} & \geq\left\|T_{g, \varphi} f_{\varphi(b)}\right\| \|_{\mathcal{B}_{\mu}} \geq \sup _{z \in B} \mu(|z|)\left|\Re\left(T_{g, \varphi} f_{\varphi(b)}\right)(z)\right| \\
& \geq \mu(|b|)|\Re g(b)|\left(\ln \frac{2}{1-|\varphi(b)|^{2}}\right)^{1 / q}
\end{aligned}
$$

From the last inequality we get the desired result.

Theorem 6. Assume that $p>1,1 / p+1 / q=1$ and $n+1+\alpha=0$, $g \in H(B), \varphi$ is a holomorphic self-map of $B$ and $\mu$ is a normal function on $[0,1)$. Then $T_{g, \varphi}: A_{\alpha}^{p} \rightarrow \mathcal{B}_{\mu}$ is compact if and only if $g \in \mathcal{B}_{\mu}$ and

$$
\lim _{|\varphi(z)| \rightarrow 1} \mu(|z|)|\Re g(z)|\left(\ln \frac{2}{1-|\varphi(z)|^{2}}\right)^{1 / q}=0 .
$$

Proof. Suppose that (25) holds. In this case, the proof of Theorem 2 still works with minor changes and therefore the details are omitted.

Conversely, suppose that $T_{g, \varphi}: A_{\alpha}^{p} \rightarrow \mathcal{B}_{\mu}$ is compact, then it is clear that $T_{g, \varphi}: A_{\alpha}^{p} \rightarrow \mathcal{B}_{\mu}$ is bounded. It follows from the proof of Theorem 5 that $g \in \mathcal{B}_{\mu}$. Let $\left(z_{k}\right)_{k \in \mathbb{N}}$ be a sequence in $B$ such that $\left|\varphi\left(z_{k}\right)\right| \rightarrow 1$ as $k \rightarrow \infty$. 
Set

$$
f_{k}(z)=\left(\ln \frac{2}{1-\left|\varphi\left(z_{k}\right)\right|^{2}}\right)^{-1 / p}\left(\ln \frac{2}{1-\left\langle z, \varphi\left(z_{k}\right)\right\rangle}\right), k \in \mathbb{N}
$$

Using Theorem 1.12 of [16], we see that $\left(f_{k}\right)_{k \in \mathbb{N}}$ is a bounded sequence in $A_{\alpha}^{p}$. Moreover, $f_{k} \rightarrow 0$ uniformly on compact subsects of $B$. It follows from Lemma 3 that $\left\|T_{g, \varphi} f_{k}\right\|_{\mathcal{B}_{\mu}} \rightarrow 0$ as $k \rightarrow \infty$. Since

$$
\begin{aligned}
\left\|T_{g, \varphi} f_{k}\right\|_{\mathcal{B}_{\mu}} & =\sup _{z \in B} \mu(|z|)\left|\Re\left(T_{g, \varphi} f_{k}\right)(z)\right| \\
& \geq \mu\left(\left|z_{k}\right|\right)\left|\Re g\left(z_{k}\right)\right|\left(\ln \frac{2}{1-\left|\varphi\left(z_{k}\right)\right|^{2}}\right)^{1 / q},
\end{aligned}
$$

we obtain

$$
\lim _{k \rightarrow \infty} \mu\left(\left|z_{k}\right|\right)\left|\Re g\left(z_{k}\right)\right|\left(\ln \frac{2}{1-\left|\varphi\left(z_{k}\right)\right|^{2}}\right)^{1 / q}=0,
$$

from which we get the desired result.

Theorem 7. Assume that $p>1,1 / p+1 / q=1$ and $n+1+\alpha=0$, $g \in H(B), \varphi$ is a holomorphic self-map of $B$ and $\mu$ is a normal function on $[0,1)$. Then $T_{g, \varphi}: A_{\alpha}^{p} \rightarrow \mathcal{B}_{\mu, 0}$ is bounded if and only if $T_{g, \varphi}: A_{\alpha}^{p} \rightarrow \mathcal{B}_{\mu}$ is bounded and $g \in \mathcal{B}_{\mu, 0}$.

Proof. Suppose that $T_{g, \varphi}: A_{\alpha}^{p} \rightarrow \mathcal{B}_{\mu, 0}$ is bounded, then $T_{g, \varphi}: A_{\alpha}^{p} \rightarrow \mathcal{B}_{\mu}$ is bounded. Taking $f(z)=1$, then employing the boundedness of $T_{g, \varphi}$ : $A_{\alpha}^{p} \rightarrow \mathcal{B}_{\mu, 0}$, we get $g \in \mathcal{B}_{\mu, 0}$, as desired.

Conversely, suppose that $T_{g, \varphi}: A_{\alpha}^{p} \rightarrow \mathcal{B}_{\mu}$ is bounded and $g \in \mathcal{B}_{\mu, 0}$. For each polynomial $p(z)$,

$$
\mu(|z|)\left|\Re\left(T_{g, \varphi} p\right)(z)\right|=\mu(|z|)|p(\varphi(z))||\Re g(z)| \leq\|p\|_{\infty} \mu(|z|)|\Re g(z)| .
$$

From the above inequality, it follows that for each polynomial $p, T_{g, \varphi}(p) \in$ $\mathcal{B}_{\mu, 0}$. Since the set of all polynomials is dense in $A_{\alpha}^{p}$, for every $f \in A_{\alpha}^{p}$ there is a sequence of polynomials $\left(p_{k}\right)_{k \in \mathbb{N}}$ such that $\left\|p_{k}-f\right\|_{A_{\alpha}^{p}} \rightarrow 0$ as $k \rightarrow \infty$. From the boundedness of $T_{g, \varphi}: A_{\alpha}^{p} \rightarrow \mathcal{B}_{\mu}$, we have that

$$
\left\|T_{g, \varphi} p_{k}-T_{g, \varphi} f\right\|_{\mathcal{B}_{\mu}} \leq\left\|T_{g, \varphi}\right\|\left\|p_{k}-f\right\|_{A_{\alpha}^{p}} \rightarrow 0, \quad \text { as } k \rightarrow \infty .
$$

From this and since $\mathcal{B}_{\mu, 0}$ is a closed subset of $\mathcal{B}_{\mu}$, we obtain

$$
T_{g, \varphi} f=\lim _{k \rightarrow \infty} T_{g, \varphi} p_{k} \in \mathcal{B}_{\mu, 0} .
$$

Therefore $T_{g, \varphi}: A_{\alpha}^{p} \rightarrow \mathcal{B}_{\mu, 0}$ is bounded. The proof is completed. 
Using Theorems 6 and 7, similarly to the proof of Theorem 4, we obtain the following result. We omit the proof.

Theorem 8. Assume that $p>1,1 / p+1 / q=1$ and $n+1+\alpha=0$, $g \in H(B), \varphi$ is a holomorphic self-map of $B$ and $\mu$ is a normal function on $[0,1)$. Then $T_{g, \varphi}: A_{\alpha}^{p} \rightarrow \mathcal{B}_{\mu, 0}$ is compact if and only if

$$
\lim _{|z| \rightarrow 1} \mu(|z|)|\Re g(z)|\left(\ln \frac{2}{1-|\varphi(z)|^{2}}\right)^{1 / q}=0 .
$$

From Theorems 5-8, we have the following corollary.

Corollary 2. Suppose $p>1,1 / p+1 / q=1$ and $n+1+\alpha=0$. Let $g \in H(B)$ and $0<\beta<\infty$. Then the following statements hold.

(i) $T_{g}: A_{\alpha}^{p} \rightarrow \mathcal{B}^{\beta}$ is bounded if and only if $T_{g}: A_{\alpha}^{p} \rightarrow \mathcal{B}_{0}^{\beta}$ is bounded if and only if

$$
\sup _{z \in B}\left(1-|z|^{2}\right)^{\beta}|\Re g(z)|\left(\ln \frac{2}{1-|z|^{2}}\right)^{1 / q}<\infty ;
$$

(ii) $T_{g}: A_{\alpha}^{p} \rightarrow \mathcal{B}^{\beta}$ is compact if and only if $T_{g}: A_{\alpha}^{p} \rightarrow \mathcal{B}_{0}^{\beta}$ is compact if and only if

$$
\lim _{|z| \rightarrow 1}\left(1-|z|^{2}\right)^{\beta}|\Re g(z)|\left(\ln \frac{2}{1-|z|^{2}}\right)^{1 / q}=0 .
$$

Next we consider the case of $0<p \leq 1$.

Theorem 9. Assume that $n+1+\alpha=0$ and $0<p \leq 1, g \in H(B), \varphi$ is a holomorphic self-map of $B$ and $\mu$ is a normal function on $[0,1)$. Then $T_{g, \varphi}: A_{\alpha}^{p} \rightarrow \mathcal{B}_{\mu}$ is bounded if and only if $g \in \mathcal{B}_{\mu}$.

Proof. Suppose that $g \in \mathcal{B}_{\mu}$. For an $f \in A_{\alpha}^{p}$, by Lemma 1 we have

$$
\begin{aligned}
\sup _{z \in B} \mu(|z|)\left|\Re\left(T_{g, \varphi} f\right)(z)\right| & =\sup _{z \in B} \mu(|z|)|f(\varphi(z))||\Re g(z)| \\
& \leq C\|f\|_{A_{\alpha}^{p}} \sup _{z \in B} \mu(|z|)|\Re g(z)| .
\end{aligned}
$$

From the above inequality we see that $T_{g, \varphi}: A_{\alpha}^{p} \rightarrow \mathcal{B}_{\mu}$ is bounded.

Conversely, suppose that $T_{g, \varphi}: A_{\alpha}^{p} \rightarrow \mathcal{B}_{\mu}$ is bounded. Taking $f(z)=1$, then using the boundedness of $T_{g, \varphi}: A_{\alpha}^{p} \rightarrow \mathcal{B}_{\mu}$, we get the desired result.

Theorem 10. Assume that $n+1+\alpha=0$ and $0<p \leq 1, g \in H(B), \varphi$ is a holomorphic self-map of $B$ and $\mu$ is a normal function on $[0,1)$. Then 
$T_{g, \varphi}: A_{\alpha}^{p} \rightarrow \mathcal{B}_{\mu}$ is compact if and only if $g \in \mathcal{B}_{\mu}$ and

$$
\lim _{|\varphi(z)| \rightarrow 1} \mu(|z|)|\Re g(z)|=0 .
$$

Proof. Suppose that $g \in \mathcal{B}_{\mu}$ and that (32) holds. In this case, the proof is similar to the proof of Theorem 2 and hence we omit it.

Conversely, suppose that $T_{g, \varphi}: A_{\alpha}^{p} \rightarrow \mathcal{B}_{\mu}$ is compact. Then it is clear that $T_{g, \varphi}: A_{\alpha}^{p} \rightarrow \mathcal{B}_{\mu}$ is bounded. It follows from Theorem 9 that $g \in \mathcal{B}_{\mu}$. Let $\left(z_{k}\right)_{k \in \mathbb{N}}$ be a sequence in $B$ such that $\left|\varphi\left(z_{k}\right)\right| \rightarrow 1$ as $k \rightarrow \infty$. Set

$$
f_{k}(z)=\frac{1-\left|\varphi\left(z_{k}\right)\right|^{2}}{1-\left\langle z, \varphi\left(z_{k}\right)\right\rangle}, k \in \mathbb{N} .
$$

From Theorem 6.6 of [16] we see that $\left(f_{k}\right)_{k \in \mathbb{N}}$ is a bounded sequence in $A_{\alpha}^{p}$. Moreover, $f_{k}$ converges to zero uniformly on compact subsects of $B$. In view of Lemma 3 it follows that

$$
\limsup _{k \rightarrow \infty}\left\|T_{g, \varphi} f_{k}\right\|_{\mathcal{B}_{\mu}}=0
$$

On the other hand, we have

$$
\left\|T_{g, \varphi} f_{k}\right\|_{\mathcal{B}_{\mu}}=\sup _{z \in B} \mu(|z|)\left|\Re\left(T_{g, \varphi} f_{k}\right)(z)\right| \geq \mu\left(\left|z_{k}\right|\right)\left|\Re g\left(z_{k}\right)\right| .
$$

Combining (33) with (34) we see that (32) holds. The proof is completed.

Theorem 11. Assume that $n+1+\alpha=0$ and $0<p \leq 1, g \in H(B), \varphi$ is a holomorphic self-map of $B$ and $\mu$ is a normal function on $[0,1)$. Then the following statements are equivalent.

(i) $T_{g, \varphi}: A_{\alpha}^{p} \rightarrow \mathcal{B}_{\mu, 0}$ is bounded;

(ii) $T_{g, \varphi}: A_{\alpha}^{p} \rightarrow \mathcal{B}_{\mu, 0}$ is compact;

(iii) $g \in \mathcal{B}_{\mu, 0}$.

Proof. (ii) $\Rightarrow(i)$. This implication is obvious.

(i) $\Rightarrow$ (iii). Taking $f(z)=1$ and employing the boundedness of $T_{g, \varphi}: A_{\alpha}^{p} \rightarrow \mathcal{B}_{\mu, 0}$ we get that $g \in \mathcal{B}_{\mu, 0}$.

(iii) $\Rightarrow\left(\right.$ ii). Suppose that $g \in \mathcal{B}_{\mu, 0}$. For any $f \in A_{\alpha}^{p}$ with $\|f\|_{A_{\alpha}^{p}} \leq 1$, we have

$$
\mu(|z|)\left|\Re\left(T_{g, \varphi} f\right)(z)\right| \leq C\|f\|_{A_{\alpha}^{p}} \mu(|z|)|\Re g(z)| \leq C \mu(|z|)|\Re g(z)|,
$$


from which we obtain

$$
\lim _{|z| \rightarrow 1} \sup _{\|f\|_{A_{\alpha}^{p}} \leq 1} \mu(|z|)\left|\Re\left(T_{g, \varphi} f\right)(z)\right| \leq C \lim _{|z| \rightarrow 1} \mu(|z|)|\Re g(z)|=0 .
$$

Using Lemma 2 we see that $T_{g, \varphi}: A_{\alpha}^{p} \rightarrow \mathcal{B}_{\mu, 0}$ is compact and the assertion follows.

From Theorems 9-11, we obtain the following corollary.

Corollary 3. Suppose $0<p \leq 1$ and $n+1+\alpha=0$. Let $g \in H(B)$ and $0<\beta<\infty$. Then the following statements hold.

(i) $T_{g}: A_{\alpha}^{p} \rightarrow \mathcal{B}^{\beta}$ is bounded if and only if $g \in \mathcal{B}^{\beta}$;

(ii) $T_{g}: A_{\alpha}^{p} \rightarrow \mathcal{B}^{\beta}$ is compact if and only if $T_{g}: A_{\alpha}^{p} \rightarrow \mathcal{B}_{0}^{\beta}$ is bounded if and only if $T_{g}: A_{\alpha}^{p} \rightarrow \mathcal{B}_{0}^{\beta}$ is compact if and only if $g \in \mathcal{B}_{0}^{\beta}$.

2.3. Case $n+1+\alpha<0$.

Theorem 12. Assume that $p>0$ and $n+1+\alpha<0, g \in H(B), \varphi$ is a holomorphic self-map of $B$ and $\mu$ is a normal function on $[0,1)$. Then the following statements are equivalent.

(i) $T_{g, \varphi}: A_{\alpha}^{p} \rightarrow \mathcal{B}_{\mu}$ is bounded;

(ii) $T_{g, \varphi}: A_{\alpha}^{p} \rightarrow \mathcal{B}_{\mu}$ is compact;

(iii) $g \in \mathcal{B}_{\mu}$.

Proof. (ii) $\Rightarrow(i)$. It is obvious.

$(i) \Rightarrow($ iii $)$. Taking $f(z)=1$, then using the boundedness of $T_{g, \varphi}: A_{\alpha}^{p} \rightarrow$ $\mathcal{B}_{\mu}$ we get the desired result.

(iii) $\Rightarrow\left(\right.$ ii). Suppose that $g \in \mathcal{B}_{\mu}$. For an $f \in A_{\alpha}^{p}$, by Lemma 1 we see that $f$ is continuous on the closed unit ball and so is bounded in $B$. Therefore

$$
\mu(|z|)\left|\Re\left(T_{g, \varphi} f\right)(z)\right|=\mu(|z|)|f(\varphi(z))||\Re g(z)| \leq C\|f\|_{A_{\alpha}^{p} \mu(|z|)|\Re g(z)| .}
$$

From the above inequality we see that $T_{g, \varphi}: A_{\alpha}^{p} \rightarrow \mathcal{B}_{\mu}$ is bounded. Let $\left(f_{k}\right)_{k \in \mathbb{N}}$ be any bounded sequence in $A_{\alpha}^{p}$ and $f_{k} \rightarrow 0$ uniformly on $\bar{B}$ as $k \rightarrow \infty$. We have

$$
\left\|T_{g, \varphi} f_{k}\right\|_{\mathcal{B}_{\mu}}=\sup _{z \in B} \mu(|z|)\left|f_{k}(\varphi(z)) \Re g(z)\right| \leq\|g\|_{\mathcal{B}_{\mu}} \sup _{z \in B}\left|f_{k}(\varphi(z))\right| \rightarrow 0,
$$

as $k \rightarrow \infty$. Employing Lemma 4 , the implication follows.

Theorem 13. Assume that $p>0$ and $n+1+\alpha<0, g \in H(B), \varphi$ is a holomorphic self-map of $B$ and $\mu$ is a normal function on $[0,1)$. Then the following statements are equivalent. 
(i) $T_{g, \varphi}: A_{\alpha}^{p} \rightarrow \mathcal{B}_{\mu, 0}$ is bounded;

(ii) $T_{g, \varphi}: A_{\alpha}^{p} \rightarrow \mathcal{B}_{\mu, 0}$ is compact;

(iii) $g \in \mathcal{B}_{\mu, 0}$.

Proof. The proof is similar to the proof of Theorem 11 and therefore we omit the details.

From Theorems 12 and 13, we get the following corollary.

Corollary 4. Suppose $p>0$ and $n+1+\alpha<0$. Let $g \in H(B)$ and $0<\beta<\infty$. Then the following statements hold.

(i) $T_{g}: A_{\alpha}^{p} \rightarrow \mathcal{B}^{\beta}$ is bounded if and only if $T_{g}: A_{\alpha}^{p} \rightarrow \mathcal{B}^{\beta}$ is compact if and only if $g \in \mathcal{B}^{\beta}$;

(ii) $T_{g}: A_{\alpha}^{p} \rightarrow \mathcal{B}_{0}^{\beta}$ is bounded if and only if $T_{g}: A_{\alpha}^{p} \rightarrow \mathcal{B}_{0}^{\beta}$ is compact if and only if $g \in \mathcal{B}_{0}^{\beta}$.

Acknowledgements. The author would like to thank the anonymous referee for pointing out errors in the first version of this paper and for valuable comments and suggestions.

\section{References}

[1] D. C. Chang, S. Li and S. Stević, On some integral operators on the unit polydisk and the unit ball, Taiwanese J. Math. 11 (2007), 1251-1286.

[2] C. C. Cowen and B. D. MacCluer, Composition Operators on Spaces of Analytic Functions, CRC Press, Boca Raton, 1995.

[3] H. Hedenmalm, B. Korenblum and K. Zhu, Theory of Bergman Spaces, Springer-Verlag, New York, 2000.

[4] Z. Hu, Extended Cesàro operators on mixed norm spaces, Proc. Amer. Math. Soc., 131 (2003), 2171-2179.

[5] Z. Hu, Extended Cesàro operators on the Bloch space in the unit ball of $\mathbb{C}^{n}$, Acta Math. Sci. Ser. B Engl. Ed., 23 (2003), 561-566.

[6] Z. Hu, Extended Cesàro operators on Bergman spaces, J. Math. Anal. Appl., 296 (2004), 435-454.

[7] S. Li, Volterra composition operators between weighted Bergman space and Bloch type spaces, J. Korea Math. Soc., 45 (2008), 229-248.

[8] S. Li, Riemann-Stieltjes operators from $F(p, q, s)$ to Bloch space on the unit ball, Vol. 2006, Article ID 27874, J. Inequal. Appl. (2006), 14 pages. 
[9] S. Li and S. Stević, Compactness of Riemann-Stieltjes operators between $F(p, q, s)$ and $\alpha$-Bloch spaces, Publ. Math. Debrecen, 72 (2008), 111-128.

[10] S. Li and S. Stević, Riemann-Stieltjes operators on Hardy spaces in the unit ball of $\mathbb{C}^{n}$, Bull. Belg. Math. Soc. Simon Stevin, 14 (2007), 621-628.

[11] K. Madigan and A. Matheson, Compact composition operators on the Bloch space, Trans. Amer. Math. Soc., 347 (1995), 2679-2687.

[12] S. Ohno, K. Stroethoff and R. Zhao, Weighted composition operators between Bloch-type spaces. Rocky Mountain J. Math. 33 (2003), 191215.

[13] S. Stević, On an integral operator on the unit ball in $\mathbb{C}^{n}$, J. Inequal. Appl., 1 (2005), 81-88.

[14] J. Xiao, Riemann-Stieltjes operators on weighted Bloch and Bergman spaces of the unit ball, J. London. Math. Soc., 70 (2004), 199-214.

[15] R. Zhao and K. Zhu, Theory of Bergman spaces on the unit ball, Mem. Soc. Math. France., to appear.

[16] K. Zhu, Spaces of Holomorphic Functions in the Unit Ball, SpringerVerlag, New York, 2005.

Department of Mathematics

JiaYing University

514015, Meizhou, GuangDong

China

(E-mail : jyuzxl@163.com) 


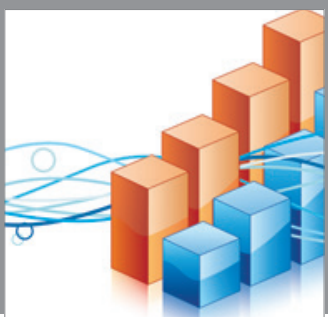

Advances in

Operations Research

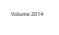

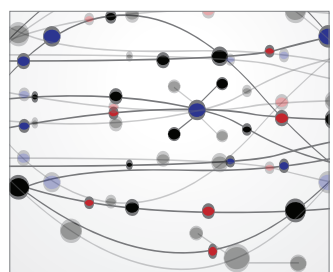

\section{The Scientific} World Journal
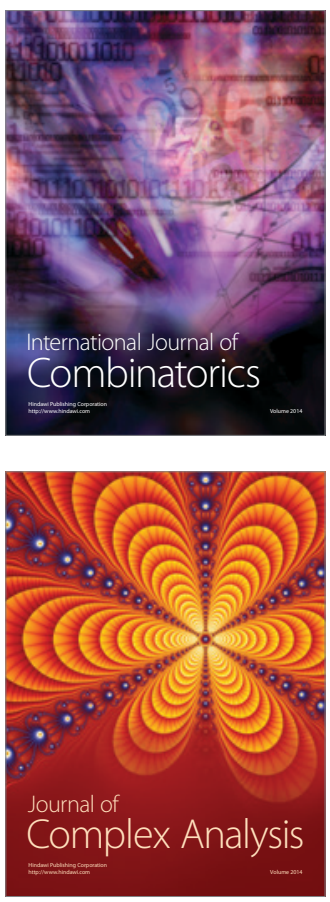

International Journal of

Mathematics and

Mathematical

Sciences
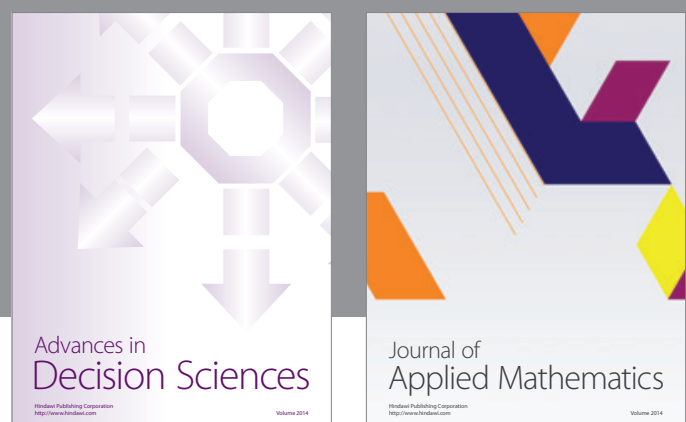

Journal of

Applied Mathematics
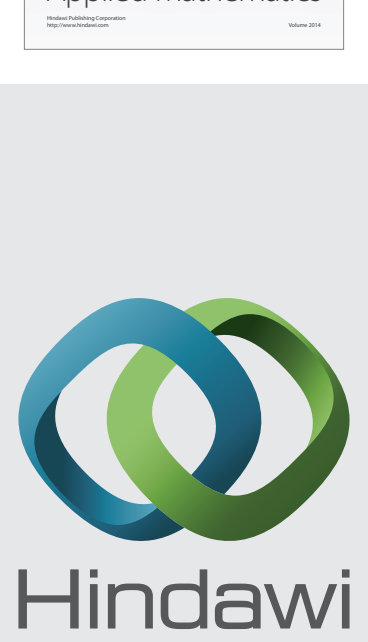

Submit your manuscripts at http://www.hindawi.com
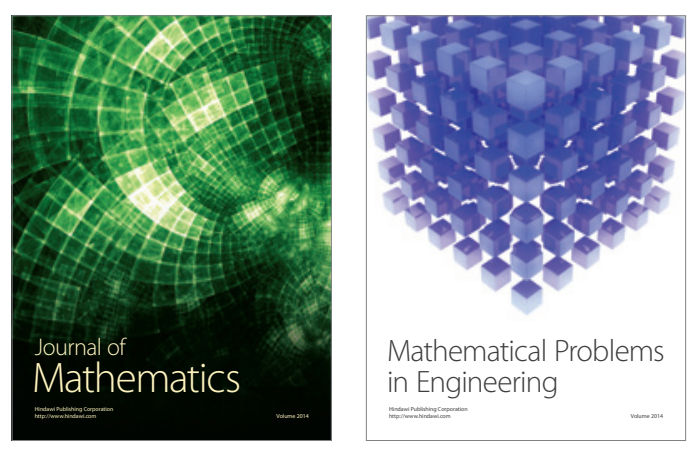

Mathematical Problems in Engineering
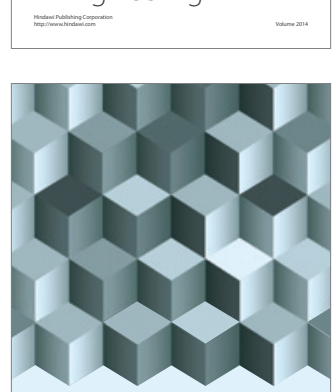

Journal of

Function Spaces
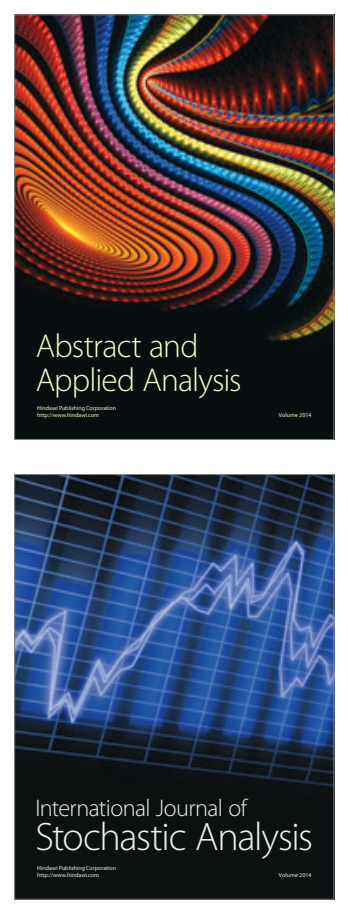

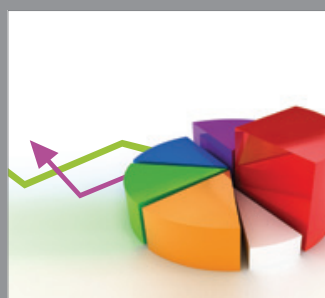

ournal of

Probability and Statistics

Promensencen
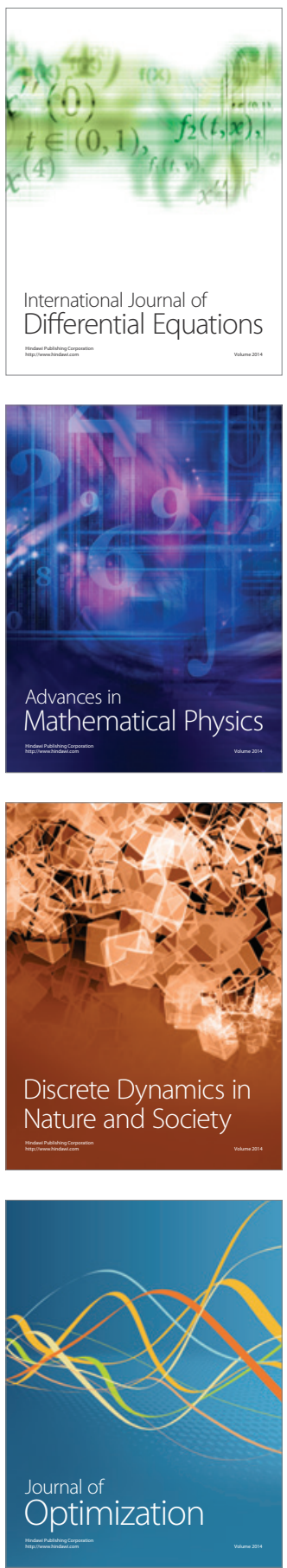